\title{
i-Gel Supraglottic Airway for Inexperienced Anesthesia Residents
}

\author{
Mariko Senda, Tokujiro Uchida, Hiroyuki Kobinata, Koichi Nakazawa, Koshi Makita \\ Department of Anesthesiology, Graduate School of Medicine, Tokyo Medical and Dental University, Tokyo, Japan. \\ Email: uchida.mane@tmd.ac.jp
}

Received January $6^{\text {th }}, 2013$; revised February $3^{\text {rd }}, 2013$; accepted February $28^{\text {th }}, 2013$

\begin{abstract}
i-gel (Intersurgical Ltd., Wokingham, UK) is a new supraglottic airway device with a cuff made of thermoplastic elastomer gel. We retrospectively studied easiness of insertion and troubles in ventilation when this device was used by less experienced anesthesiologists. Consecutive eleven cases undergoing surgery under general anesthesia were studied. i-gel was successfully inserted in 7 cases (63\%) at the first attempt, 2 cases at the second attempt without changing the size. In one case, the size was changed at the second attempt, which resulted in the successful insertion. There was one case of failed insertion even at the second attempt. Fiberoptic observation of the glottis showed direct contact of the cuff to the arytenoids cartilage in cases with successful insertion, however, spastic glottis, defined as contact of vocal cords was observed in 4 cases, and down-folding of the epiglottis was observed in 2 cases. During maintenance of anesthesia, ventilation trouble was observed in 3 cases. We conclude that i-gel should only be used with sufficient knowledge, preparations, skills or support from experienced physician to solve problems related to the safe use of supraglottic airway devices.
\end{abstract}

Keywords: Supraglottic Airway Device; i-Gel; Airway Management

\section{Introduction}

i-gel (Intersurgical Ltd., Wokingham, UK) is a new supraglottic airway device with a cuff made of thermoplastic elastomer gel. Because of its unique design, the insertion technique is thought to be easy regardless of experience, allowing quick insertion without air leakage even under positive pressure ventilation [1-4]. For verification of these characteristics, we retrospectively studied ease of insertion and problems with ventilation when this device was used by less-experienced anesthesia residents. Although the insertion times in successful cases were comparable with previous studies, we observed a lower success rate at the first attempt, and ventilation troubles were observed in several cases during the maintenance of anesthesia. In this report, we characterize the problems with i-gel when it is used by inexperienced anesthesia residents.

\section{Case Descriptions}

Consecutive eleven cases in which i-gel was inserted by anesthesia residents were retrospectively selected for this case report. All the procedures were performed as routine clinical procedures and informed consent was obtained from all the patients for all the anesthetic management including the use of i-gel by the residents under the supervision of senior anesthesiologists. i-gel insertion and maintenance of anesthesia were carried out by six anesthesia residents who had experienced supraglottic airway devices less than 20 times. They all read an instruction manual and watched an instruction video provided by the manufacturer, and then were trained in the insertion technique using a mannequin before attempting its use in patients. The size was determined by the patient's body weight as described in the manufacture's instructions; (size 3 for 30 - $60 \mathrm{~kg}$, size 4 for 50 - $90 \mathrm{~kg}$, and size 5 for above $90 \mathrm{~kg}$ ). General anesthesia was induced with propofol (1.5 - $3.5 \mathrm{mg} / \mathrm{kg}$ ) supplemented with fentanyl (1 - 2 $\mu \mathrm{g} / \mathrm{kg}$ ) or remifentanil $(0.05 \mu \mathrm{g} / \mathrm{kg} / \mathrm{min})$. Muscle relaxant was not used on the first attempt for all cases, and rocuronium could be used from the second attempt based on the decision of the anesthesiologists in charge of each case. i-gel was inserted according to the manufacturer's instructions, and the insertion time, defined as the time from the end of mask ventilation to the commencement of ventilation through i-gel, was routinely recorded in each anesthesia record. After i-gel was inserted and successful ventilation was confirmed, a fiberoptic view of the glottis was observed from the proximal end of the airway lumen of i-gel before the operation. 
Patient's characteristics are summarized in Table $\mathbf{1}$. The results of insertion trials are summarized in Figure 1. i-gel was successfully inserted in seven cases (63\%) on the first attempt, and two cases on the second attempt without changing the size. In one case, the size was changed from size 3 to size 4 on the second attempt, which resulted in successful insertion. There was one case of failed insertion even on the second attempt, in which an LMA-ProSeal ${ }^{\mathrm{TM}}$ (LMA North America, San Diego, CA) was finally inserted. Muscle relaxant was only given in one case in which muscle relaxation was required for successful insertion on the second attempt. In ten cases with successful insertion, the median insertion time was 24 seconds (range 10 - 91 seconds). Fiberoptic observation of the glottis showed direct contact of the tip of the cuff to the arytenoids cartilage or wedged into the hypopharynx in cases with successful insertion (Figure 2(A)). However, spastic glottis, defined as contact of the vocal cords (Figure 2(B)), was observed in four cases (36\%), and down-folding of the epiglottis (Figure 2(C)) was observed in two cases (18\%).

During maintenance of anesthesia, elevation of the airway pressure and concomitant tidal volume reduction were observed in three cases (30\% of successfully-inserted cases at anesthetic induction). Two of them showed spastic glottis, and one showed down-folding of the epiglottis on fiberoptic observation of the larynx before operation. In these cases, adjustment of the position of igel resulted in resolution in one case, while administration of fentanyl $(100 \mu \mathrm{g})$ improved ventilation in another. In the third case, the i-gel was removed and the trachea was intubated.

\section{Discussion}

Because of their design for easy insertion, various suprag-

Table 1. Characteristics of patients.

\begin{tabular}{ccc}
\hline (n = 11, Male/Female $=3 / 8)$ & & \\
\hline Age (years old) & Median & Range \\
Body weight (kg) & 52 & $29-70$ \\
Duration of anesthesia (min) & 147 & 43.8 - 84.0 \\
Duration of surgery (min) & 99 & 55 - 195 \\
Surgery & 14 - 152 \\
Mastectomy & (Number of patients) \\
Breast phyllodes tumor resection & 6 \\
Debridement and skin graft for the foot & 1 \\
Hernioplasty & 1 \\
Cervical conization & 1 \\
Transurethral resection of bladder tumor & 1 \\
\hline
\end{tabular}

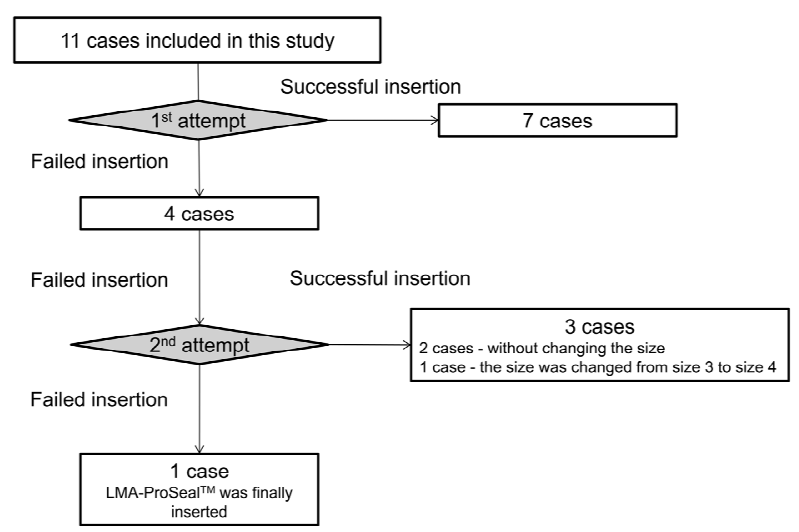

Figure 1. Summary of insertion trials.

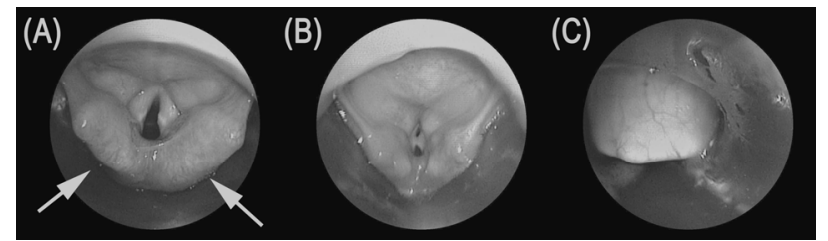

Figure 2. Representative images of fiberoptic observation of the glottis via i-gel. (A) Representative view after successful insertion. The cuff of i-gel (arrow) directly contacted with the arytenoids cartilage. (B) Spastic glottis, defined as contact of the vocal cords. (C) Down-folding of the epiglottis by the cuff of i-gel.

lottic airway devices have been studied for use by inexperienced physicians. Previous studies involving novice trainees demonstrated the median insertion time and success rate on the first attempt for the LMA-Classic ${ }^{\mathrm{TM}}$ to be $32 \mathrm{~s}$ to be $80 \%$ [5], the LMA-ProSeal ${ }^{\mathrm{TM}} 53 \mathrm{~s}$ and $73 \%$ [6], and i-gel $18 \mathrm{~s}$ and 83\% [4]. These previous results indicate that the i-gel can be placed faster and more successfully than the other devices. In the current study, the median time of insertion was 23 seconds in successful first attempts, which was comparable with this previous study. However, the rate of unsuccessful first attempts (36\%) was not negligible. Two factors may be considered from our observation. First, there is a possibility of inappropriate positioning of any supraglottic airway device. In the current study, down-folding of the epiglottis was observed in two cases (18\%) and ventilation trouble was experienced in one of those patients. Despite its unique design, called "epiglottis blocker", down-folding of the epiglottis could not be completely avoided. Although we could not conclude direct causality, attention should be paid to down-folding of the epiglottis as a possible cause of ventilation troubles. Secondly, inexperienced personnel might fail to keep sufficient anesthetic depth. We experienced four cases of spastic larynx in fiberoptic observation, and ventilation troubles were experienced in two of them. In these cases, insufficient anesthesia might be a possible factor explaining the ventilation problems. 
Because i-gel is designed to fit the larynx tightly and i-gel itself could stimulate the larynx, deeper anesthesia or use of neuromuscular blocking agents should have been considered in such cases. Although those two factors are common to all supraglottic airway devices and it is difficult to compare their frequencies because of the small size of our study population, it is important to educate inexperienced anesthesia residents to prevent these possible problems when they use i-gel. Considering these results, we should consider possible problems in airway management by i-gel when it is inserted by inexperienced anesthesia residents, and i-gel should only be used with sufficient knowledge, preparations, skills or support from experienced physician to solve problems related to the safe use of supraglottic airway devices. Because the size of the study population was too small to analyze the problems in detail, a larger scale study will be essential in establishing the safe use of this unique supraglottic airway device.

\section{REFERENCES}

[1] J. J. Gatward, T. M. Cook, C. Seller, J. Handel, T. Simpson, V. Vanek and F. Kelly, "Evaluation of the Size 4 i-Gel Airway in One Hundred Non-Paralysed Patients," Anaesthesia, Vol. 63, No. 10, 2008, pp. 1124-1130. doi:10.1111/j.1365-2044.2008.05561.x
[2] W. J. Shin, Y. S. Cheong, H. S. Yang and T. Nishiyama, "The Supraglottic Airway i-Gel in Comparison with ProSeal Laryngeal Mask Airway and Classic Laryngeal Mask Airway in Anaesthetized Patients," European Journal of Anaesthesiology, Vol. 27, No. 7, 2010, pp. 598-601. doi:10.1097/EJA.0b013e3283340a81

[3] L. G. Theiler, M. Kleine-Brueggeney, D. Kaiser, N. Urwyler, C. Luyet, A. Vogt, R. Greif and M. M. Unibe, "Crossover Comparison of the Laryngeal Mask Supreme and the i-Gel in Simulated Difficult Airway Scenario in Anesthetized Patients,” Anesthesiology, Vol. 111, No. 1, 2009, pp. 55-62. doi:10.1097/ALN.0b013e3181a4c6b9

[4] N. M. Wharton, B. Gibbison, D. A. Gabbott, G. M. Haslam, N. Muchatuta and T. M. Cook, "i-Gel Insertion by Novices in Manikins and Patients,” Anaesthesia, Vol. 63, No. 9, 2008, pp. 991-995. doi:10.1111/j.1365-2044.2008.05542.x

[5] M. G. Tan, E. R. Chin, C. S. Kong, Y. H. Chan and P. C. Ip-Yam, "Comparison of the Re-Usable LMA Classic and Two Single-Use Laryngeal Masks (LMA Unique and SoftSeal) in Airway Management by Novice Personnel," Anaesth Intensive Care, Vol. 33, No. 6, 2005, pp. 739743.

[6] N. S. Klaver, K. Kuizenga, A. Ballast and V. Fidler, “A Comparison of the Clinical Use of the Laryngeal Tube S and the ProSeal Laryngeal Mask Airway by First-Month Anaesthesia Residents in Anaesthetised Patients,” Anaesthesia, Vol. 62, No. 7, 2007, pp. 723-727. doi:10.1111/j.1365-2044.2007.05073.x 